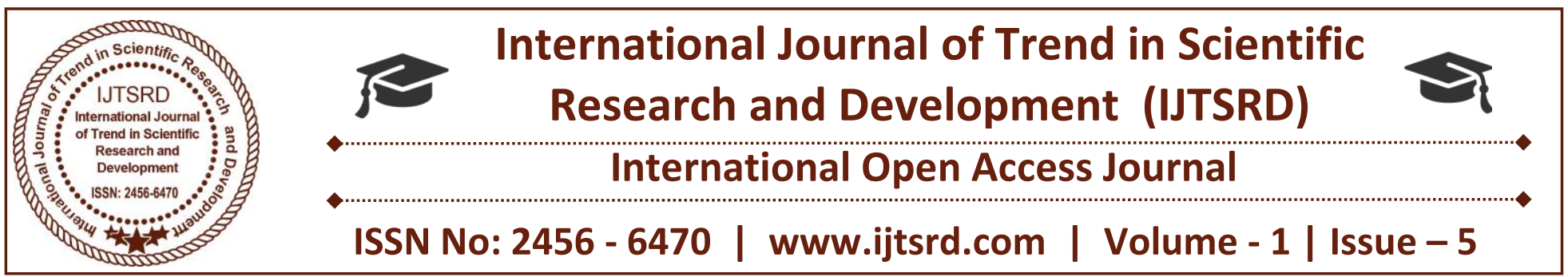

\title{
An Application of the Universal Design in Integrated Living Arrangements for Elderly People in Thailand
}

\author{
Supakit Yimsrual \\ Assistant Professor, Department of Architecture, \\ Faculty of Architecture, Naresuan University, Phitsanulok, Thailand
}

\begin{abstract}
In Thailand, the environment suitability of the integrated housing for older people is still limited. Thus, this research aimed to apply the concept of Universal Design in the study of the suitability of integrated living arrangements for the elderly and stipulate the design of integrated living arrangements for the elderly in Thailand. The data was collected from the elderly residing in the government's centers for development of social welfare for the elderly. They were 106 participants with four age ranges: 6064 years, 65-69 years, 70-74 years, and 75 or above.The study used three types of tests: behavioral interviews and the need for space and utensils, tests on the use of space and utensils, and records of participant behavior. The tests on the use of space andutensils included ten apparatuses: handrails, bathroom fittings, electrical switches, power sockets, door knobs, handles and bolts, stairs, ramps, interior and exterior floors. The results showed that a handrail should be at least $4.5 \mathrm{~cm}$. in diameter and should be at

$\mathrm{cm}$. from the floor. Door knobs or level-type knobs should be used because of their ease of use and ease to close with low physical effort. On the contrary, the use of handles and bolts should be avoided.The rise of stair should have a height of about $13 \mathrm{~cm}$. and not less than $47 \mathrm{~cm}$. for the run to accommodate the use of the elderly. The ramp should have a slope ratio of not less than 1: 12.Interior and exterior floors must be covered with a non-slip surface so that the elderly can easily walk or use a wheelchair. According to the need for space, the elderly needed space about 1.44 sq.m. per person for the rest areas. Whereas, sleeping areas should be no less than 12 sq.m. per person. The bathroom should have free space, without obstructions, of at least $1.50 \mathrm{x} 1.50 \mathrm{~m}$. The study concluded that the seven principles of the Universal Design can be applied to assess the suitability of the environment for the integrated housing of the elderly and to design an integrated housing design for the elderly appropriately.
\end{abstract} least $5 \mathrm{~cm}$. from the wall and not less than $12 \mathrm{~cm}$ from the mounting point. The bathroom should be equipped with multiple handrails to help stabilize the elderly. The wall handrail should be horizontal or L-shaped. The non-wall handrail should be folding wallmounted or T-shape floor-mounted. The sanitary ware used should be a flat sanitary ware with a height of about $400 \mathrm{~mm}$. The faucet should be a lever type faucet because it was easy to use and it needed less physical effort to turn. Electrical switches should be installed at a height of $120 \mathrm{~cm}$. from floor level while power sockets should be installed at a height of 90
Keywords: Elderly, Integrated Living, Universal Design

\section{INTRODUCTION}

It is important to have a study in the design of the living environment for the elderly widely overseas; however, in Thailand, research on such issues, especially study the suitability of the environment for integrated housing of the elderly is relatively limited. Most recent research have aimed to study the design of a specific area such asbathroom, the kitchen, the 
bedroom, and etc., while some focused on the environment of single or residential dwellings or specific purposes for example design education to prevent accidents occurring to elderly people in the residence, design of landscape and parks that are suitable for the elderly.However, the results of the study and design standards conducted overseas cannot be applied to integrated housing design for the elderly in Thailand because of the differences in physiologies, behaviors and habitats although self-reliance among the elderly in Thailand tends to vividly increase. Yet, the studies of Trairat Jaruthat et al. (2013, 2005) entitled "A Guide to Organizing Friendly Facilities for the Elderly" and "Minimum Standards for Housing and the Environment of the Elderly "have different purposes from other recent research, and they are projected to be used as references to standards for design guidelines for residential housing for the elderly in Thailand. Thus, the knowledge from these two prominent researches was applied in this study. In recent years, behavior in the use of space, as well as the demand for housing in Thailand, has shifted from a single dwelling to an integrated dwelling, such as a condominium or elderly care facilities and an elderly home (Siripanich, 2010). Consequently, the elderly living and relying on themselves in an inappropriate environment is a major factor in the occurrence of accidents in the elderly and eventually leads to disability (Siriboon, 2003). However, there is no study to determine the appropriate integrated housing design for the elderly. Therefore, this study was conducted with the focus on the appropriateness of the integrated living environment for older people in Thailand by adopting the design concept for Universal Design to assess suitability and design guidelines for integrated housing design for the elderly. It aimed to determine the minimum standards or design requirements, with an emphasis on the use of space, building equipment and behavioral responses of the elderly. The results of the research can be used to determine guidelines for designing integrated housing for older people in Thailand in the near future.

\section{RESEARCH OBJECTIVES}

This study had three-fold objectives:

1. To study the suitability of the integrated living environment for the elderly in Thailand,

2. To apply the design concept of Universal Design to design integrated housing for the elderly in Thailand, and
3. To establish guidelines of integrated housing design for the elderly in Thailand.

\section{SCOPE OF THE RESEARCH PROJECT}

\section{Scope of Case Study and Sample Population}

The research was conducted from the Government Center for Development of Social Welfare for the Elderly due to the variety of residential types, such as single detached housing and integrated housing, etc. In this study, a sample group of the elderly was recruited based on the classification system of the elderly, according to the National Statistics Office of Thailand. The subjects included 106 volunteers divided into four age groups: 60-64 years, 65-69 years, 69-74 years, and 75 years or above.

\section{RESEARCH METHODOLOGY}

The current research was a survey research that collected data from the Government Center for Development of Social Welfare for the Elderly. It included

1. An exploration of the elderly's behaviors of utilizing space and equipment,

2. An investigation of housing and living environment, sizes and locations of equipment installations relative to the physiology of the elderly, and

3. In-depth interviews with the elderly to assess how suitable the housing environment is.

The results from the surveyswere used to compare with design standards and design guidelines of Universal Design and other related documents in order to implement a test of factors affecting integrated housing designs for the elderly. The steps of data collection can be summarized as follows:

\section{Step 1 Review of Related Literature}

Documentary research and literature review will analyze the content of relevant international and national research, such as measurement of the elderly's body size, living conditions and types of the elderly's residence, ideas related to environment arrangements and housing design standards of the elderly, as well as Universal Design.

\section{Step 2 Survey of the Physical Condition of the Residence}

Survey of the physical condition of the integrated residence of the elderly included surveying the usable 
area and the equipment installation stage. The survey results would be used to compare with the design standards. It is also used as a basis for interviewing behavioral and occupational needs in building space and equipment.

\section{Step 3 In-depth Interview}

Interviews of the elderly participants consisted of both closed-ended and open-ended questions. Results from these interviews were screened to assess behavioral conditions, daily routines, living styles, and demands for space and equipment, and the results from the assessment were used to create a test of space applications and building equipment.

\section{Step 4: Test of Space and Building Equipment}

At this stage, the participants were tested regarding the use of space and equipment made by the researcher to assess the suitability of the space and the size of the utilized area, size of equipment and the installation phase of building equipment that respond to behaviors and daily routines. To obtain the aforementioned information, the researcher interviewed and observed the utilization behavior of the participants.

\section{Step 5: Analysis of Research Data}

The data obtained from this research were compared with various design standards, as well as experts' perspectives in order to summarize the results and establish guidelines for integrated housing design for the elderly in Thailand.

\section{Research Tools}

Three research tools were used in the data collection:

1. Behavioral interviews regarding daily routines and needs for the use of space and equipment,

2. A test of space and building equipment utilization, and

3. A record of behaviors in using building space and equipment.

\section{A. Behavioral Interviews}

The behavioral interview was aimed at screening and assessing the behaviors and routines of the elderly. Its structure was divided into two parts:

Part 1: Basic information about the participants which included,

1. Number of participants divided by residence types, age groups, and health,
2. Daily activities and exercise, and

3. Living behaviors, the useof building space and equipment.

Part 2: Information about the housing environment requirements which is divided into three main parts:

1. Characteristics of housing and length of residence,

2. Opinions on the environment facilities and services in residential area, and

3. Needs of building space and equipment.

\section{B: The Test of Building Space and Equipment}

The test of building space and equipment was used to explore the appropriateness of the space and the size of the active area, the size and the installation phase of building equipment used by the elderly in everyday life. The test builds on seven design principles of the Universal Design: there are equitable use, flexibility in use, simple and intuitive use, perceptible information, tolerance for error, low physical effort, and size and space for approach and use. The details are as follows:

Part 1: A test of the use of outdoor and indoor space included:

1. Bedrooms,

2. Leisure areas,

3. Bathrooms,

4. Walkways, stairs, ramp and interior floors, and

5. Walkways, stairs, ramp and exterior floors.

Part 2: A test of building equipment which included:

1. Handrails, both general handrails and bathroom handrails, taking into account of the size, level of installation and its materials,

2. Faucet,

3. Electric switches, based on the type and level of installation,

4. Powersockets, based on the type and level of installation,

5. Doors and windows,

6. Door knobs, handles and bolts, and

7. Toilet bowl.

In addition, the building equipment used in the test was certified for industrial products and it was commercially available in the local market.

\section{C: A Record and Observation of the Use of Building Space and Equipment}

The purpose of these tools executed simultaneously was to record the participants' behaviors of using building space and equipment. 


\section{RESULTS}

The results of this study showed that the environment of government centers for the elderly's social welfare development was in accordance with the requirement standards of appropriate and safe environment for the elderly suggested by Bureau of Environmental Health of Thailand in 2010 and the ministerial regulation on building facilities for the disabled and the elderly launched by Department of Empowerment of Persons with Disabilities in2005. The suitable housing for them was single-floor housing. If having more floors is compulsory, a vertical commuting system and ramps with a slope ratio of 1:12 are obligatory in order to accommodate the wheelchair use of the elderly with physical movement limitations. In addition, separated rooms in residential buildings for the elderly to live together should accommodate not more than two persons per room. Furthermore, an integrated room should accommodate not more than four older persons, whereas a sleeping building may provide lodgings to not more than eight persons, fourbeds per row. The survey results regarding the need to use the sleeping area by the elderly showed that each elderly person needs about 12 sq.m. of sleeping area, approximately 1.44 sq.m. of recreation area and $1.50 \times 1.50 \mathrm{~m}$. of a bathroom with clear interior space and an easy access. Thus, the study of the use of interior and exterior space can be summarized as follows.

\section{A: Outdoor Space}

Elderly people needed space for exercise and other recreational activities. They were a collaborative activity that demonstrates the need for social participation. Therefore, the design of the area must be easily accessible and accommodate the use of the elderly with varying limitations of movement, sensory perceptions. In this study, the composition of the outdoor space was collected from the elderly who tested the risers and the runs of the stair, the slope of the ramps, and footpath surfaces. The results are summarized as follows.

\section{The height of the risers}

The study found that the height of the risers appropriate for the pace of the elderly is at an altitude of $130 \mathrm{~mm}$, which is less than the altitude of the risers set in the design standard, which regulates that the height of the risers should not be more than $150 \mathrm{~mm}$. The result is due to the elderly's physiological differentiation and shorter walking behavior (i.e. a lower height, a narrower width of foot separation and a shorter raise of the legs while walking).

\section{The length of the runs}

Based on the results of the study, it was found that the elderly walked up the stairs by using an apt foot, followed by the other foot to allocate weight. It is characterized by discontinuous walking. Therefore, the width of stair steps must be wider than normal to increase foot rest, distribute the weight and determine the pace of the next step. It should be at least $510 \mathrm{~mm}$. wide to support the use of the elderly who use a walker or other walking aids.

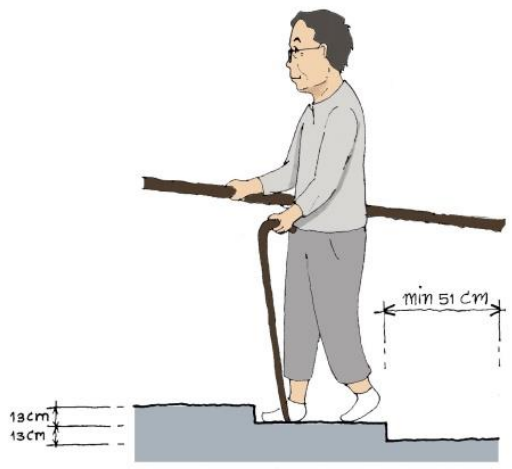

Figure 1: A sketch of the risers and the runs

\section{Ramp slope}

To test the gradient of the ramp, the elderly were asked to walk up and down the ramp, as well as using a wheelchair. Most of the elderly participants chose the slope at 1: 8 ratio of the ramp slope, and at a ratio of 1: 12 in the case of using a wheelchair. These ratios correspond to the slope gradient of the design standard, which specifies that the length of a ramp should be $6,000 \mathrm{~mm}$. or less. In cases where the ramp length exceeds $6,000 \mathrm{~mm}$, a minimum of $1,500 \mathrm{~mm}$. rest area should be provided between the ramp sections.

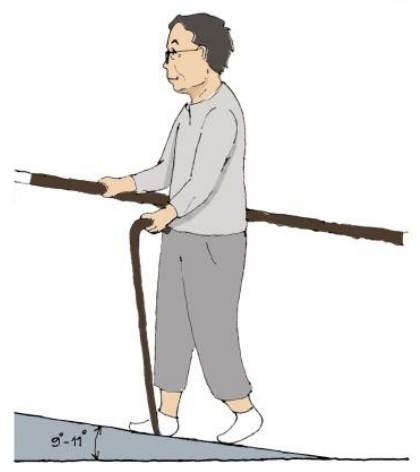

Figure 2: A sketch of the ramp inclination 


\section{Various surface types}

The surface tested was divided into different categories according to its use. The elderly testedfloors of four surface types: washed sand, nonslip tile, washed gravel and paving block. It has been found that most elderly chose washed-sand surfaces and non-slip tile floors because they were the surfaces that helped them feel safe and comfortable to walk on. For suitable areas for work, terrace or balcony, patio area, fitness area, outdoor pedestrian areas, the elderly chose a washed-gravel floor because its rough and non-slip texturemade them feel safe in walking. For the footpath, the elderly chose a paving block. The results of this study are in line with the design standards in that the surface texture for the elderly must be made with non-slip materials.

\section{B: Interior Space}

According to studies, it has been found that the elderly spend most of the day ( 80 percent) inside the building, in the common areas, followed by the bedroom. Daily activities that the elderly liked most were recreational activities and leisure activities, which require an integrated space, separated from a living space, of approximately 1.44 sq.m. per person, and a sleeping area of 12 sq.m. per person, together with a bed of $40 \mathrm{~cm}$. in height and at least a bedside space of at least $90 \mathrm{~cm}$. in width. This was required for their walking aid or wheelchair. The survey found that common accidents in the elderly, especially older people aged 75 and over, were tripping falls and stair falls. Thus, the interior space must not be levelled. If it was necessary to have different floor levels, a staircase or ramp should be provided with a range and size that were appropriate for the mobility behavior of the elderly. It should also include a sturdy handrail on both sides, for example, the height of a space between stair riser of $130 \mathrm{~mm}$. and a stair run with 510 millimeter width. The suitable materials for indoor floors were wood, laminated materials, artificial wood and non-slip tiles. The results regarding the use of building components by the elderly can be summarized as follows.

\section{Handrails}

According to the international design standards for the elderly, the corridors, toilets, common areas and rooms need to be equipped with handrails or other auxiliary equipment (Rednier, 2002). Based on the results of the handle test, older people chose a $45 \mathrm{~mm}$. diameter grip which is larger than the design standard, as they viewed that it was easier to handle and it seemed stronger. Furthermore, handrail material must be smooth, non-slip, stable, strong, and safe to grasp, and the height of the handrails must fit the physique of the elderly. A proper measurement from the ground level to the top level of the grip for mounting a handrail should be $800 \mathrm{~mm}$., which corresponds to the design standard. In the case of wall mounting, the distance from the wall rail should not be less than 50 $\mathrm{mm}$., and the height of the handrail from the wallmounting point must not be less than $120 \mathrm{~mm}$. In addition, the wall of the rail should be smooth to facilitate the catch and allow the elderly to move their hands on the rail without obstacles.

\section{Handrails in the bathroom}

The study found that the elderly participants chose a bathroom handrail that was made of smooth material, strong, stable, harmless to handle and non-slip. For optimum height, the handrails in the bathroom should be installed at an altitude of $900 \mathrm{~mm}$. from the floor, which is higher than the design standard. The handrail should be at least $4.5 \mathrm{~cm}$. in diameter and should be at least $5 \mathrm{~cm}$. from the wall and not less than $12 \mathrm{~cm}$. from the wall mounting point. A bathroom should have handrails in several sides to help the elderly balancethemselves. The wall handrails should be horizontal or L-shaped while the non-wall ones should have a folding wall mount or a T-shaped floor mount.

\section{Faucet}

The test results showed that the side-mounted faucet was suitable for most applications because it could be opened and closed easily with little effort. This may be because the elderly have less ability to use hands effectively. The result goes in line with the international design standards in that all bathroom fixtures must be fully leveraged so that the elderly can open or close without clutching a hand.

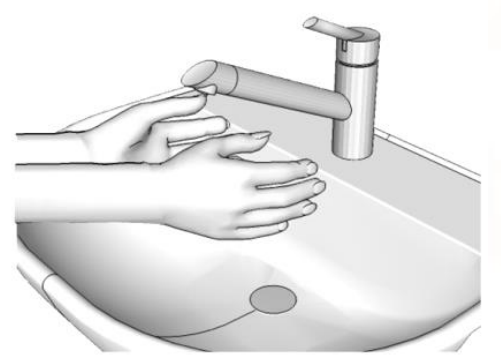

Figure 3: A sketch of the side-mounted faucet 


\section{Electric switch and power socket}

The test results showed that the level of the switch that was appropriate to the behavior of the elderly should be about 120 centimeters from the ground floor. The level of the appropriate power outlet should be about 90 centimeters from the ground level. An electrical switch appropriate for the elderly's use should have a large open-close key for easy operation, and the power plug should be equipped with a power cut switch.

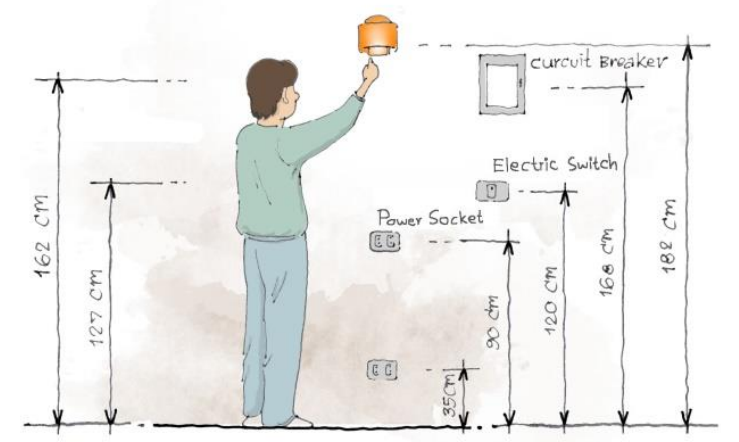

Figure 4: A proper level of electric switch and power socket

\section{Doors - windows}

Doors and windows suitable for the elderly should be sliding doors and sliding windows. The door should be at least 80 centimeters wide. The window should be at least 60 centimeters wide. Door handles should be a catch rod, leveraged or spindle because of their ease of use and low closing force.

6. Knobs, door handles and latches.

The results demonstrated that the elderly chose to use the handle with a twist or push rod because of less force used to open or close. Similarly, a grooved door knob was preferred because it is uneven and can easily be caught or rotated. For proper installation levels, a door knob should be mounted between 100 and $120 \mathrm{~cm}$. in height from the floor level. Door bolts should be avoided for the safety of the elderly in cases where the elderly cannot help themselves or where they have an accident.

\section{Toilet bowl}

Based on a survey of the physiology of the male elderly persons, the height from the floor to the toilet seat is about $401 \mathrm{~mm}$.and about $370 \mathrm{~mm}$. in height for the female elderly persons. Therefore, a toilet for the elderly should have a toilet seat with a backrest at an altitude of about $400 \mathrm{~mm}$. from the floor level, which is below the level set in the design standard (i.e., 450
- $500 \mathrm{~mm}$.).This may be because Thai older people have a smaller body than older people in the west.

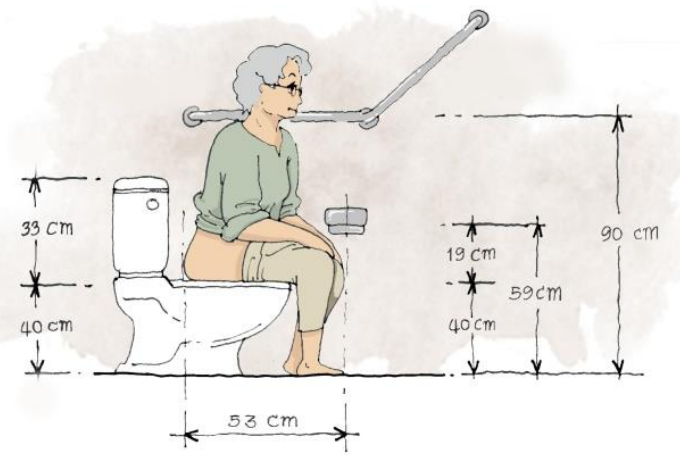

Figure 5: A proper size of toilet bowl

\section{CONCLUSIONS AND RECOMMENDATION}

The results from this study can besummarized as follows. The information provided by the research, particularly the needs of space utilization, size and installation phase of building equipment, differed from the standards in some parts due to the physiological differentiation of the elderly. For example, the size of handrails that the elderly needed was larger than the size specified in the standard and the standard altitude of the toilet bowl from the floor level to the toilet seat is set to be between 450 and 500 $\mathrm{mm}$., but the Thai elderly required the altitude of between $370-400 \mathrm{~mm}$, etc.

Although the information was taken merely from a group of older people, it indicated that the design standards which are the foreign knowledge cannot be applied in the design of all elderly residents in Thailand. In addition, the data from the research reflects the habits of living and the use of building accessories for the elderly in the living environment with which they have long been familiar; thus, some building equipment, which did not comply with the design criteria or standards but which were familiar to the Thai elderly, were chosen by the elderly. For instance, a grooved door knob was chosen due to its uneven surface and its ability to handle or rotate is similar to a twist-type handle or a push rod, which is in accordance with standards used in many countries. The Universal Design to specify design guidelines for equipment in integrated residence of the elderly can be properly applied according to seven criteria:

1. Equitable use

2. Flexibility in use

3. Simple and intuitive use

4. Perceptible information 
5. Tolerance for error

6. Low physical effort

7. Size and space for approach and use

The research recommended that the future study should cover the elderly wit $\mathrm{h}$ different physical and living conditions, including the comparison of body physiology of Thai elderly in different age ranges in order to study the factors affecting the design of the living environment for elderly people. The research should also compare the elderly's physiological data before and after use of equipment in question. In addition, it should compare the design criteria and standards that is legally required or standardized to determine a guideline for the design of residences for the elderly in Thailand.

\section{REFERENCES}

1) Bureau of Environmental Health of Thailand (2010). Standards of Safe Environment for the Elderly. Bangkok.

2) Chayawan N. and Nodel J. (2000). Housing and Advocacy by Families of the Thai Elderly.Bangkok: College of Population Studies, Chulalongkorn University.

3) Department of Empowerment of Persons with Disabilities (2005). Ministerial Regulation on Building Facilities for the Disabled and the Elderly. Bangkok.

4) Jaruthat T. et al. (2013). A Handbook of Agile Facilities for the Elderly.Bangkok. ChulalongKorn University.

5) Jaruthat T. et al. (2005). Minimum Standards for a Project of Housing and Environment for the Elderly. Bangkok. Chulalongkorn University.

6) RednierV. (2002). Assisted Living Housing by Design. New York. Van NostrandReihold.

7) Siriboon S. (2003). Response to Problems and Needs of the Elderly. Case Study of Establishing a Social Service Center for the Elderly. Bangkok. Demographics College, Chulalongkorn University.

8) Siripanich B. (2010). Thai Elderly People. Bangkok. Mor Chao Baan Publishing. 